\title{
Roles of Oxygen Vacancy in Improper Ferroelectrics
}

Shiqing Deng ${ }^{1,2}$, Shaobo Cheng ${ }^{3}$, Changsong Xu ${ }^{4}$, Binghui $\mathrm{Ge}^{5}$, Xuefeng Sun ${ }^{6}$, Rong Yu ${ }^{1}$, Wenhui Duan $^{4}$, Yimei $\mathrm{Zhu}^{2}$ and Jing $\mathrm{Zhu}^{1}$

1. National Center for Electron Microscopy in Beijing, School of Materials Science and Engineering, Tsinghua Univ., Beijing, P. R. China.

2. Dept. of Condens. Matter Phys. and Mater. Sci., Brookhaven National Lab., Upton, NY.

3. Dept. of Mater. Sci. and Eng., Canadian Centre for Electron Microscopy, McMaster Univ., Hamilton, ON, Canada.

4. Dept. of Phys. \& State Key Lab. of Low-Dimens. Quantum Phys., Tsinghua Univ., Beijing, P. R. China.

${ }^{5}$ Beijing Natl. Lab. for Condens. Matter Phys., Inst. of Phys., Chin. Acad. of Sci., Beijing, P. R. China.

6. Hefei National Lab. for Physical Sciences at Microscale, Univ. of Science and Technology of China, Anhui, P. R. China.

Multiferroics have attracted much attention due to the strong coupling between ferroelectricity and ferromagnetism, which can lead promising applications in multifunctional devices [1]. Accompanied with robust ferroelectricity $\left(\mathrm{T}_{\mathrm{C}} \sim 900 \mathrm{~K}\right)$ and low-temperature antiferromagnetism $\left(\mathrm{T}_{\mathrm{N}} \sim 70 \mathrm{~K}\right)$ [2], hexagonal $\mathrm{RMnO}_{3}(\mathrm{R}=$ rare-earth elements) shows its potential as a single-phase multiferroic material. Such a system is known for the novel improper ferroelectric mechanism that generates superior characteristics, including insensitivity to depolarization field [3]. This improper ferroelectricity comes from opposing dipoles caused by opposite displacement of yttrium ions at two symmetry inequivalent sites and is associated with tilting and distortion of $\mathrm{MnO}_{5}$ bipyramids [3,4]. Therein, specific oxygen atoms play a vital role in the interaction between the nontrivial trimerization of $\mathrm{MnO}_{5}$ bipyramids and the displacement of yttrium [4,5], which determines the nature of improper ferroelectricity and the topologically protected domain structure [6]. Questions then arise as to how improper ferroelectricity would be altered by the existence of oxygen vacancies and what the effects are for oxygen vacancies at different symmetry sites. Answers to these questions can be helpful for understanding the underlying atomic mechanism of improper ferroelectricity and allowing tuning coupling effects between ferroic orders.

Here, we report the critical roles of oxygen vacancies at different symmetry sites in modulating improper ferroelectricity in hexagonal $\mathrm{YMnO}_{3}$. Combining atomic resolved high-angle annual dark field scanning transmission microscope (HAADF-STEM) images and electron energy loss spectra (EELS), magnitudes of ferroelectric polarization in both stoichiometric and oxygen-deficient areas are extracted based on precisely measured yttrium positions. The latter shows remarkable polarization deterioration, which depends on the concentration of oxygen vacancies. Using $a b$ initio density functional theories (DFT) calculations, we find that such phenomena originate from the significantly decreased strength of hybridizations between $\mathrm{O}-2 p$ and $\mathrm{Y}-4 d_{z^{2}}$ orbitals when oxygen vacancies, especially in-plane oxygen vacancies, are created. Furthermore, eight possible vacancy configurations are constructed and calculated to obtain variations of polarization magnitudes with various oxygen vacancy concentrations. The ferroelectric polarization shows a monotonous decrease with the increase of oxygen vacancy concentration. Our results verify the importance of $\mathrm{O}-2 p$ and $\mathrm{Y}-4 d_{z^{2}}$ hybridizations in driving improper ferroelectricity in hexagonal manganites and provide a possible tuning strategy. The insights from this work that the oxygen vacancy can be considered as an atomic multiferroic tuning element suggest the new thinking of material design and implementation [7].

References: 
[1] S. Dong et al, Adv. Phys. 64 (2015), p. 519.

[2] S. B. Cheng et al, Adv. Funct. Mater. 26 (2016), p. 3589.

[3] N. Sai et al, Phys. Rev. Lett. 102 (2009), p. 107601.

[4] S. Q. Deng et al, ACS Appl. Mater. Interfaces 9 (2017), p. 27322.

[5] S. B. Cheng et al, Appl. Phys. Lett. 106 (2015), p. 062905.

[6] S. Q. Deng et al, J. Am. Ceram. Soc. 100 (2017), p. 2373.

[7] The authors acknowledge funding from Chinese National Natural Science Foundation $(11374174,51390471$, 51527803), National 973 Project of China (2015CB654902), and National Key Research and Development Program (2016YFB0700402).
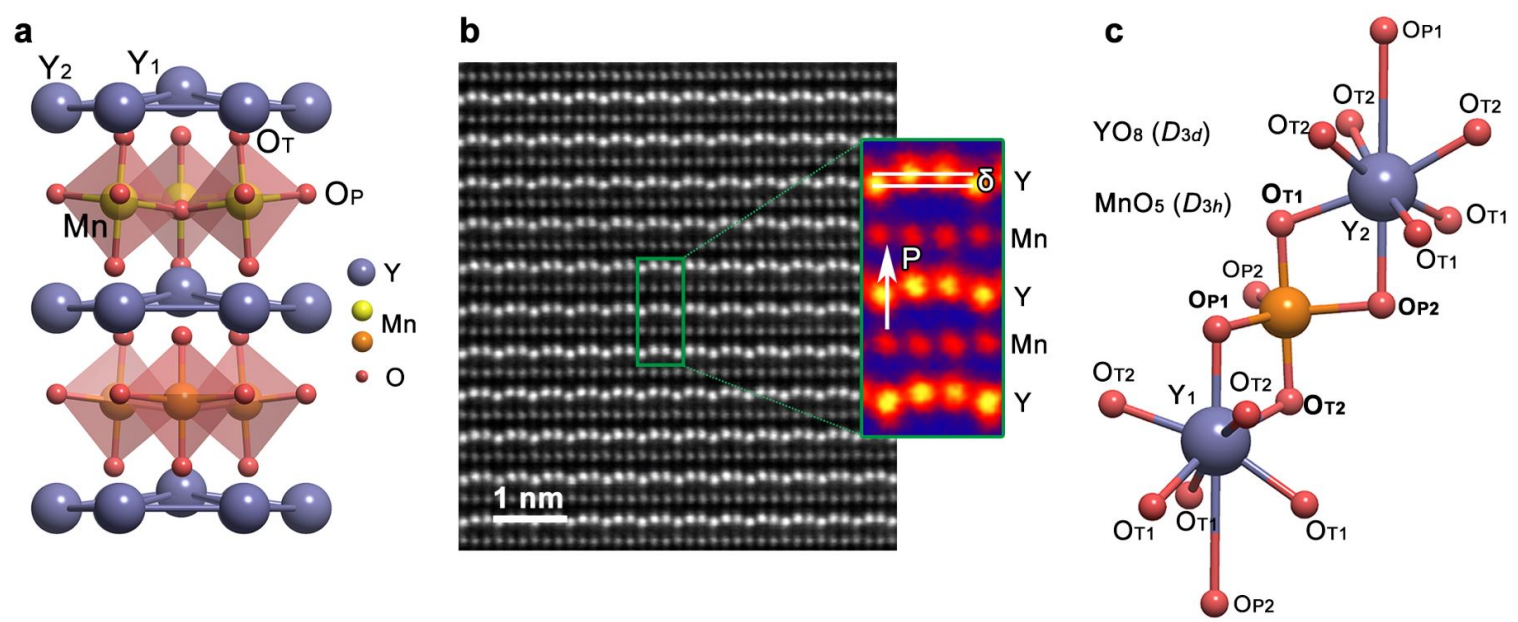

Figure 1. Atomic structure of hexagonal $\mathrm{YMnO}_{3}$. (a) Crystal model. (b) A typical HAADF-STEM image, showing up-updown lattice periodicity of $\mathrm{Y}$, which indicates upward ferroelectric polarization. $\mathrm{Y}$ displacement is marked by $\delta$ in the inset. (c) $\mathrm{YO}_{8}$ and $\mathrm{MnO}_{5}$ cages including oxygen atoms at different symmetry sites.
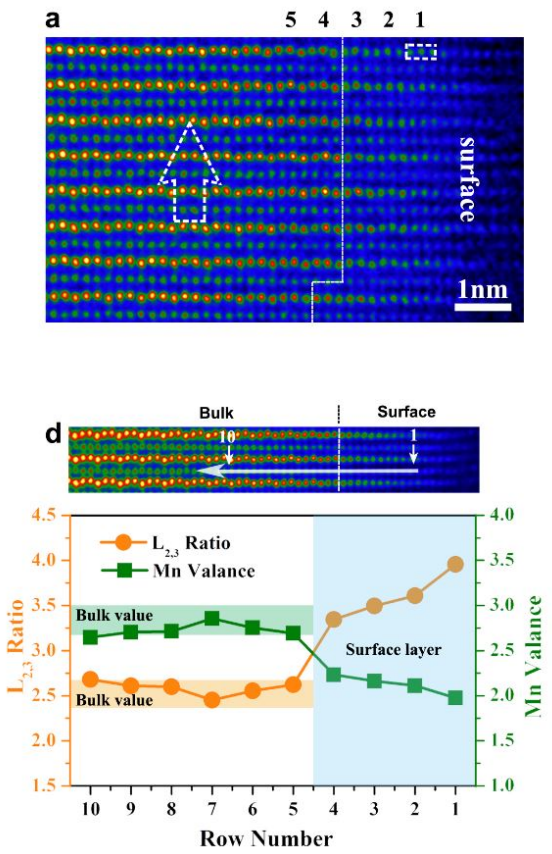
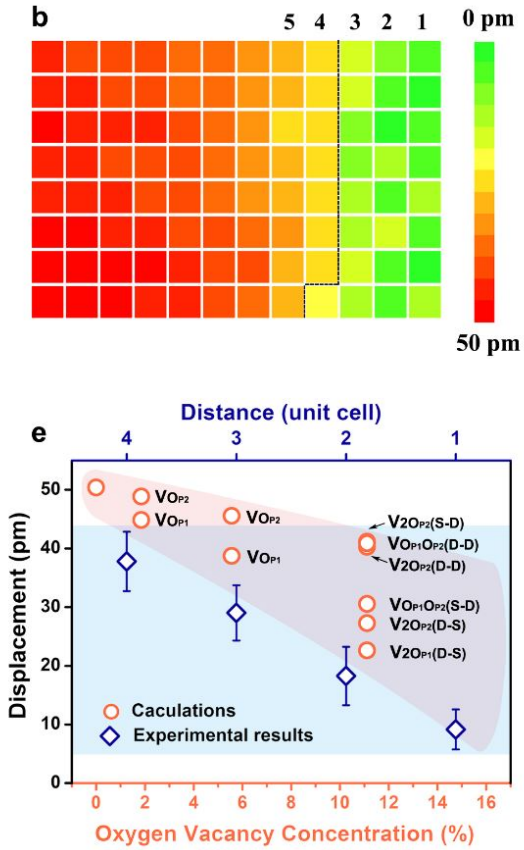
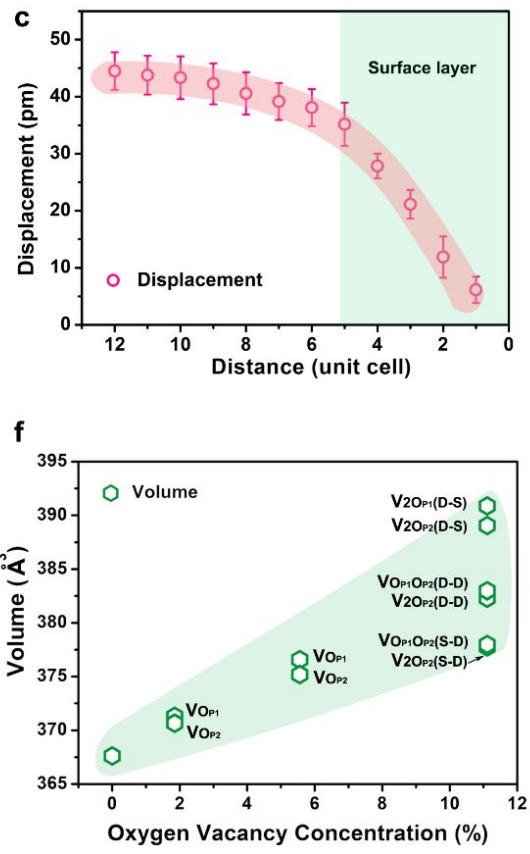

Figure 2. Dependence of ferroelectric polarizations on oxygen vacancies. (a) A HAADF-STEM image, showing upward polarization at bulk region (left part) and decreased $\mathrm{Y}$ displacement at surface (right part). (b) Magnitude map of $\mathrm{Y}$ displacement. (c) Average displacements as a function of distance from surface. (d) $\mathrm{L}_{2,3}$ ratios and $\mathrm{Mn}$ valence states as a function of distance marked by number, which are measured by EELS. Oxygen-deficient areas correspond to the polarization decrease areas. (e-f) Variations of polarizations (e) and unit cell volumes (f) with oxygen vacancy concentration. 